Attila Péteri

Budapest

\title{
Epistemik und Korpuspragmatik ${ }^{1}$ Korpusgestützte empirische Analyse des epistemischen Hintergrundes im Diskurs
}

\author{
DOI: 10.14232/fest.bassola.19
}

\begin{abstract}
Im vorliegenden Beitrag wird eine Forschungsmethode dargestellt, mit der ausgehend von der quantitativen und qualitativen Analyse epistemischer Ausdrücke und ihrer Kombinationen in Texten die Positionen der Diskursakteure relativ zum gemeinsamen epistemischen Hintergrund sowie die dynamische Entwicklung dieses Hintergrundes erschlossen werden können. Durch die quantitative Analyse werden die epistemischen Ausdrücke in zwei Subkategorien (Faktizität behauptende und Faktizität relativierende Ausdrücke) geteilt. Anschließend wird gezeigt, wie die beiden Subkategorien innerhalb eines Textes verwendet werden und die Position unterschiedlicher Diskursakteure charakterisieren.
\end{abstract}

\section{Einstieg: Ausgangspunkt und Zielsetzungen}

Es ist allgemein bekannt, dass Diskursakteure über ein gemeinsames Wissen verfügen, das teilweise aus dem allgemeinen Weltwissen bzw. aus allgemeinen kommunikativen Erfahrungen (Textsortenwissen, Wissen über Kommunikationsstrategien usw.), teilweise aus diskursiv relevantem Wissen zum jeweiligen Diskursthema besteht. Dieses gemeinsame Wissen wird epistemischer Hintergrund genannt. Diskursbeteiligte machen ständig Annahmen über das Wissen der anderen Diskursteilnehmer und positionieren sich vor diesem epistemischen Hintergrund, und zwar häufig durch die Verwendung expliziter epistemischer Ausdrucksmittel.

\footnotetext{
Mit diesem Beitrag möchte ich dem Jubilar herzlich danken für die viele Unterstützung, Konsultationen und für die Möglichkeit der Zusammenarbeit im EuroGr@mm-Projekt.
} 
Busse (2008) sieht die eigentliche Aufgabe der Diskurslinguistik in der adäquaten Beschreibung dieses epistemischen Hintergrundes und setzt Diskurslinguistik mit Epistemologie gleich. Diese Neuorientierung der Forschung bedeutet ihm zufolge keineswegs den Verzicht auf die genaue Untersuchung sprachlicher Formen und Strukturen. Die Frage „Was ist die Bedeutung dieser Form?" wird jedoch als unzureichend entlarvt und durch die Frage „Wie wirkt diese Form im gegebenen Diskurszusammenhang?“ ersetzt. Durch einen Ausdruck wird immer ein ganzer Wissensrahmen evoziert, der diskursive Wert dieses Ausdrucks ist nicht einfach ableitbar von seiner (wahrheits)semantischen Bedeutung, sondern hängt von seiner im gegebenen Kontext wahrgenommenen evokativen Kraft ab. ${ }^{2}$ Spitzmüller / Warnke (2008) erarbeiten eine Mehrebenenanalyse als Arbeitsmethode für die Diskurslinguistik, die von der genauen Erfassung einzelner Wortstrukturen über eine inhaltszentrierte und eine akteurzentrierte Analyse bis hin zur transtextuellen Perspektive reicht.

Im vorliegenden Beitrag wird eine konkrete Arbeitsmethode gezeigt, wie man ausgehend von der genauen Analyse sprachlicher Formen (sowohl mit quantitativen als auch mit qualitativen Untersuchungsmethoden) zur Erschließung des sich dynamisch wandelnden epistemischen Hintergrundes gelangt. Die untersuchten sprachlichen Formen sind dabei grammatikalisierte und lexikalische epistemische Ausdrücke. Das Novum der hier dargestellten Methode besteht einerseits darin, dass nicht die eng genommene sprachliche Bedeutung dieser Ausdrücke, sondern ihre evokative Kraft, ihre diskursive Leistung, den Gegenstand der Analyse bildet. ${ }^{3}$ Andererseits werden nicht die Einzelausdrücke in ihrem propositionalen Rahmen interpretiert, weil davon ausgegangen wird, dass nicht sie allein für die diskursive Leistung verantwortlich sind, sondern ihre Kombinationen in einzelnen Textteilen. Texte werden durch die Veränderung des epistemischen Hintergrundes strukturiert. Die einzelnen Textteile zeichnen sich dadurch aus, dass die dort verwendeten epistemischen Ausdrücke ein Raster bilden, einander ergänzen und den jeweiligen epistemischen Hintergrund bzw. das Verhältnis der Diskursbeteiligten dazu charakterisieren.

\footnotetext{
2 Unter, evokativer Kraft' wird verstanden, welche Wissensbestände bzw. welche Einstellungen der gegebene Ausdruck im Wissen der Diskursbeteiligten hervorruft.

3 Insofern entbehrt diese Analyse ein explizites Semantikmodell und ist pragmatisch verankert.
} 


\section{Der theoretische Hintergrund}

Als theoretischer Hintergrund gelten die einschlägigen Arbeiten Ekkehard Felders und seiner Forschergruppe in Heidelberg, die mit der vorhin erwähnten epistemologischen Forschungsrichtung gut vereinbar sind. Felder untersucht vor allem die Faktizitätsherstellung in Diskursen. Er erstellt ein diskursanalytisches Modell zur Erfassung der Entstehung diskursiven Wissens, das auch für die vorliegenden Forschungen als Ausgangspunkt gilt.

In ihrer methodologischen Studie unterscheiden Felder / Müller / Vogel (2011) drei methodologische Schwerpunkte für die empirische Diskursforschung, und zwar die

\section{- handlungsorientierte, \\ - gesellschaftsorientierte und \\ - wissensorientierte Perspektive.}

Im ersten Fall stellt man „signifikante sprachliche Muster in Teil- oder Vergleichskorpora“ (Felder / Müller / Vogel 2011: 12) fest, die für „bestimmte Begriffe, Themen und Perspektiven auf Sachverhalte" (ebenda) konstitutiv sind. Die aufgrund automatischer bzw. halbautomatischer quantitativer Methoden ausgewählten Muster für relevante Ausdrücke „dienen als Vorstrukturierung für qualitative Analysen einzelner exemplarischer Textpassagen“" (ebenda). Diese Perspektive fokussiert die sprachliche Ausdrucksebene, jedoch aus einer pragmatischen Perspektive: Nicht die Strukturen selbst stehen im Mittelpunkt, sondern ihre diskursive Wirkung. Ausgegangen wird davon, dass die Einzelstrukturen mehr oder weniger konventionalisierte Muster bilden (vgl. dazu Bubenhofer 2009). ${ }^{4}$

In der gesellschaftsorientierten Diskursforschung werden gesellschaftliche Verhältnisse „als Determinante sprachlicher Perspektivierung“ (Felder / Müller / Vogel 2011: 13) wie etwa soziale Ungleichheit, Machtverhältnisse usw. analysiert. In wissensorientierten Analysen werden schließlich diskursrelevante Wissenskonfigurationen untersucht:

$4 \quad$ Die Kategorie ,Muster ${ }^{\text {' }}$ ist nicht an eine bestimmte sprachliche Ebene gebunden. Es kann aus einzelnen Worten, grammatischen Strukturen, aus ihren Kombinationen, sogar aus der Kombination sprachlicher und nichtsprachlicher Elemente bestehen. Wesentlich ist dabei, dass ein Muster „als Vorlage für die Produktion weiterer Zeichenkomplexe dient“ (Bubenhofer 2009: 23). 
In diesem Bereich ist das korpuslinguistische Vorgehen nicht sonderlich weit verbreitet. Das erklärt sich leicht dadurch, dass Wissensbestände nur interpretativ aus Texten plausibel gemacht werden können, ein qualitativer Zugang also unumgänglich erscheint. Allerdings haben sich hier korpuslinguistische Methoden zur Vorstrukturierung und Identifizierung zentraler Diskursmuster bewährt, in denen Wissenskonfigurationen ausgedrückt sind [...]. Auch können auf diese Weise umstrittene Wissensbereiche, , agonale Zentren“ [...] identifiziert werden. (Felder / Müller / Vogel 2011: 14)

Genau an diesem Punkt soll zu dem von Felder entwickelten Modell beigetragen werden, indem eine korpuslinguistische Methode zur quantitativen Erschließung des Gebrauchs epistemischer Ausdrücke entwickelt wird, die anschließend anhand qualitativer Analysen verifiziert wird.

„Wissen ist nicht, Wissen wird gemacht.“ (Felder 2013: 13) - so lautet die Grundhypothese von Felder. Dementsprechend laufen auch die ersten beiden diskurslinguistischen Perspektiven auf die dritte, wissensorientierte, hinaus. Im Laufe des Diskurses werden Fakten hergestellt, die von den Diskurspartnern als Wissen mindestens eines Diskursbeteiligten wahrgenommen werden. Hervorzuheben ist dabei, dass der Faktbegriff bei Felder nicht mit dem alltäglichen Faktbegriff übereinstimmt, denn Fakten sind bei ihm Konstrukte, die im Laufe des Diskurses zustande gebracht werden und als solche grundsätzlich bestreitbar und perspektiviert sind: ${ }^{5}$

Fakten existieren nicht aus sich heraus, sondern sind in Form sprachlicher Zeichenketten unmittelbar an Diskursakteure, deren Macht und gesellschaftliche Rolle sowie an das Prestige der Kommunikationsorgane gebunden. (Felder 2013: 15)

\footnotetext{
Der Terminus Fakt wird bei Felder aus dem lateinischen Verb facere , machen' abgeleitet: Fakten sind notwendigerweise subjektiv. Den Objektivitätsbegriff ersetzt er mit der Kategorie der Multiperspektivität (vgl. Felder 2013: 15f.). Im Gegensatz zu den Fakten bilden die Tatsachen eine Kategorie der außersprachlichen Wirklichkeit, sie sind ontologisch begründet, bilden aber nach Felder keinen Gegenstand der linguistischen Forschung (vgl. Felder 2016: 161f.).
} 
Allgemein und intersubjektiv akzeptierte Fakten gelten bei ihm als Daten (ebenda 14f.). Im Diskurs sind notwendigerweise verschiedene Perspektiven vorhanden, die von den Diskursakteuren auch sprachlich transparent gemacht werden können. Falls in einem Diskursabschnitt mehrere, einander widersprechende Wissensinhalte miteinander konfrontiert werden, spricht Felder von sog. agonalen Zentren. Diese zeichnen sich dadurch aus, dass es keinen allgemein akzeptierten Wissensrahmen, sondern nur unterschiedliche, miteinander kämpfende Perspektiven gibt (ebenda 21). Agonale Zentren können in einem Korpus mit bestimmten Trägerausdrücken, z.B. mit konzessiven und adversativen Konnektoren identifiziert werden. Mithilfe einer Kookkurrenzanalyse dieser Trägerausdrücke „entstehen extensional geprägte onomasiologische Vernetzungen, die auf handlungsleitende Konzepte zurückgeführt werden können“ (Felder 2011: 138).

\section{Methoden und Ergebnisse der Korpusanalyse}

\subsection{Epistemische Ausdrücke im Korpus}

Am Germanistischen Institut der Eötvös-Loránd-Universität wird schon seit mehreren Jahren anhand eines umfangreichen thematisch geordneten Textkorpus $^{6}$ an halbautomatischen bzw. automatischen Methoden gearbeitet, mit denen epistemische Einstellungen des Sprechers aufgedeckt und analysiert werden können. Die empirische Analyse basiert auf vordefinierten sprachlichen Ausdrücken. Diese sind teilweise grammatikalisiert (Modalverben, Modalpartikeln), teilweise lexikalisch (z.B. epistemische Vollverben wie glauben, meinen, hoffen, befürchten, Substantive wie Meinung, Standpunkt, Skepsis, Angst, modale Satzadverbien usw.). ${ }^{7}$

\footnotetext{
6 Das sog. „Budapester Korpus“ ist ein zweisprachiges deutsch-ungarisches thematisch geordnetes Vergleichskorpus, das auf der deutschen Seite 196 Millionen Tokens in etwa 182 Tausend POS-annotierte Texte enthält. 27 Mio. Tokens davon wurden auch weiter verarbeitet, in diesem Teilkorpus sind auch epistemische Ausdrücke annotiert, so dass eine automatische quantitative Analyse möglich ist.

7 Die Auswahlmethode der relevanten Ausdrücke wurde detailliert in Péteri (2018) beschrieben.
} 


\subsection{Automatische quantitative Analyse}

Im ersten Schritt wurde eine automatische Korpusanalyse mit Hilfe eines selbst entwickelten Computerprogramms durchgeführt.

Das Programm hat die Häufigkeit des gemeinsamen Auftretens der epistemischen Ausdrucksmittel in einem Text berechnet. So hat sich herausgestellt, dass sich bestimmte Ausdrucksmittel deutlich miteinander solidarisieren und häufig gemeinsam auftreten. Die quantitative Untersuchung wurde in Péteri (2018) in allen Details behandelt. Hier soll ein Beispiel genannt werden.

Das epistemische Verb hoffen drückt eine positive Erwartung in Bezug auf die Zukunft aus, die jedoch mit einem gewissen Unsicherheitsfaktor verbunden ist. Im Gesamtkorpus verfügt hoffen über eine relative Häufigkeit von 16, berechnet auf 100000 Textwörter und gerundet. In den Texten, in denen aber auch voraussichtlich mindestens einmal vorkommt, beträgt der relative Häufigkeitswert von hoffen 24, in den Texten, die rechnen oder Erachten oder Hoffnung enthalten, beträgt dieser Wert 21, in denen mit erwarten 20, in denen mit eventuell 19, in denen mit befürchten, Einschätzung und hoffentlich 18, in denen mit wahrscheinlich 17. So lässt sich zeigen, dass Wörter, die ähnliche epistemische Einstellungen ausdrücken, häufig miteinander in einem Text kombiniert werden. Bei hoffen sind die häufigen Kontextpartner diejenigen epistemischen Ausdrücke, die eine zukunftsbezogene Schätzung bedeuten (voraussichtlich, rechnen, erwarten), aber auch allgemeine Unsicherheitsausdrücke (eventuell, Einschätzung, wahrscheinlich) werden in relativ vielen Texten mit hoffen kombiniert. Auch das negative Erwartung bedeutende Pendant, das Verb befürchten, ist ein häufiger Kontextpartner, was damit zu erklären ist, dass beide eine zukunftsbezogene Bedeutung haben und dass in vielen Texten, und zwar in agonalen Diskursen, einander widersprechende Perspektiven versprachlicht, folglich auch epistemische Ausdrücke mit gegensätzlicher Bedeutung zur Charakterisierung der unterschiedlichen Perspektiven benutzt werden.

\subsection{Unsicherheits- und Faktizitätsausdrücke}

Das von uns entwickelte Programm erstellt eine sog. Textkookkurrenztabelle, in der gut erkennbar ist, welche epistemischen Ausdrücke häufig miteinander kombiniert werden. Dadurch lassen sich diese Ausdrücke mit einer bottom- 
up-Methode auf empirischer Basis subkategorisieren. Die lexikalischen epistemischen Ausdrücke gliedern sich eindeutig in zwei Grobkategorien. Zur ersten zählen diejenigen, mit denen der Sprecher den Faktizitätswert seiner Aussage mindert, indem er seine Unsicherheit, seinen Zweifel o.Ä. ausdrückt (im Folgenden Unsicherheitsausdrücke genannt). Die häufigsten sind diejenigen, die eine allgemeine relativierende Bedeutung haben. Zu ihnen gehören zahlreiche Verben (glauben, meinen, finden, halten usw.), aber auch Substantive (Meinung, Erachten, Standpunkt usw.), Adjektive (unsicher, ungefähr, etwaig usw.) sowie Adverbien (vielleicht, kaum usw.). Außer den häufigen Wörtern, die allgemeine Unsicherheit ausdrücken, gibt es auch Wörter mit spezifischer Bedeutung, die daher seltener sind z.B. hoffen, befürchten, warnen, Angst, voraussichtlich usw.

Die zweite Gruppe bilden die faktizitätsstiftenden Ausdrücke, die den Faktizitätswert der Aussage festlegen (im Folgenden Faktizitätsausdrücke genannt). Dazu gehören einerseits Ausdrücke der subjektiv-epistemischen Sicherheit (der Sprecher weiß etwas sicher, begründet aber seine Sicherheit nicht) wie z.B. klar, deutlich, sicher, evident, unbestreitbar, unumstritten, notwendig, selbstverständlich, natürlich, andererseits Ausdrücke, die etwas als objektive Gegebenheit markieren wie etwa Fall, Fakt, Faktum, faktisch, tatsächlich, real, Realität, offensichtlich, offenbar bzw. diejenigen, die Faktizität dadurch herstellen, dass etwas festgelegt bzw. entschieden wird: mitteilen/Mitteilung, feststellen/Feststellung, behaupten/Behauptung, bestätigen/Bestätigung, festlegen/Festlegung, entscheiden/Entscheidung, zustimmen/Zustimmung, ablehnen/Ablehnung.

Die Unsicherheits- und die Faktizitätsausdrücke können auch mit einem einfachen Transformationstest voneinander unterschieden werden. Durch Unsicherheitsausdrücke wird die Geltung der Aussage eingeschränkt, sodass das Gegenteil der Aussage nicht ausgeschlossen wird. Faktizitätsausdrücke markieren demgegenüber die ausschließliche Geltung der Aussage und schließen das Gegenteil aus:

(1) Ich glaube, dass Klaus die Aufgabe gemacht hat. $\rightarrow$ Es ist möglich, dass Klaus die Aufgabe gemacht hat, es ist jedoch auch nicht unmöglich, dass er sie nicht gemacht hat.

(2) Ich stelle fest, dass Klaus die Aufgabe gemacht hat. $\rightarrow$ Es ist nicht möglich, dass Klaus die Aufgabe nicht gemacht hat. 
Die grammatikalisierten epistemischen Ausdrücke (Modalverben und Modalpartikeln) haben abstrakte Bedeutungen und lassen sich nicht in die vorliegenden Subklassen einordnen. Bestimmte Tendenzen sind jedoch sichtbar: Faktizitätsausdrücke werden häufiger mit den Modalverben müssen und sollen, Unsicherheitsausdrücke häufiger mit können, dürfen mögen und wollen kombiniert. In der Umgebung der Faktizitätsausdrücke sind Modalpartikeln ferner seltener als mit Unsicherheitsausdrücken. Modalpartikeln, die auf irgendein Gegensatzverhältnis auf der Ebene der Äußerungssituation verweisen können (doch, eben/halt, einfach, schon) sind in agonalen Diskurssituationen häufiger als zustimmende Modalpartikeln wie ja, mal. ${ }^{8}$

Die überwiegende Mehrheit der Texte enthält epistemische Ausdrücke, und zwar sowohl Unsicherheits- als auch Faktizitätsausdrücke. Die folgende Tabelle gibt eine Übersicht über die absoluten Zahlen und die Verteilung in Prozent:

\begin{tabular}{|l|c|c|}
\hline & $\begin{array}{c}\text { absolute } \\
\text { Zahl }\end{array}$ & $\begin{array}{c}\text { prozentmäßige } \\
\text { Verteilung }\end{array}$ \\
\hline Gesamtzahl der Texte im Korpus & 44.278 & $100 \%$ \\
\hline Texte ohne epistemische Ausdrücke & 5.277 & $11,91 \%$ \\
\hline Texte, die nur Unsicherheitsausdrücke enthalten & 7.986 & $18,03 \%$ \\
\hline Texte, die nur Faktizitätsausdrücke enthalten & 2.005 & $4,53 \%$ \\
\hline $\begin{array}{l}\text { Texte, die beide Typen epistemischer Ausdrücke } \\
\text { enthalten }\end{array}$ & 29.010 & $65,52 \%$ \\
\hline
\end{tabular}

Tab. 1: Verteilung der Korpustexte mit und ohne epistemische Ausdrücke

Texte ohne epistemische Ausdrücke sind meistens Kurznachrichten oder bearbeiten allgemeine Konsensthemen. Durch das Fehlen epistemischer Ausdrücke bekommen die Aussagen einen hohen Faktizitätswert. Texte, die nur Unsicherheitsausdrücke enthalten, zeugen von einem unsicheren epistemischen Hintergrund mit vielen offenen Fragen. Selten sind diejenigen Texte, die nur Faktizitätsausdrücke enthalten. Diese behandeln im Gegensatz zu den Texten ohne epistemische Ausdrücke im Allgemeinen Dissensthemen,

\footnotetext{
8 Wobei der Modalpartikelgebrauch auch von anderen Faktoren abhängt, besonders vom nähebzw. distanzsprachlichen Charakter des Textes (vgl. Ágel / Hennig 2007).
} 
in denen mehrere Perspektiven bzw. Standpunkte miteinander kontrastiert werden. Während mit Aussagen ohne jegliche epistemische Markierung einfach Fakten festgelegt werden, die von den anderen Diskursbeteiligten als gemeinsames diskursives Wissen ohne Weiteres angenommen werden, verfügen die Faktizitätsausdrücke über eine persuasive Funktion, indem der eine Diskursbeteiligte die anderen über den Faktizitätswert seiner Aussage überzeugen will. Damit wird aber zugleich unterstellt, dass die gegebene Aussage im Diskurs nicht einheitlich akzeptiert wird, sondern gerade eine Dissensperspektive darstellt.

Am häufigsten sind natürlich diejenigen Texte, in denen sowohl Unsicherheits- als auch Faktizitätsausdrücke zu finden sind. Die Faktizitätsausdrücke dienen zur Festlegung der verschiedenen Positionen. Die Unsicherheitsausdrücke werden meistens in argumentierenden Textteilen benutzt, um zu markieren, dass die Argumentationen mit diversen Unsicherheitsfaktoren verbunden sind. Das Verhältnis der Faktizitäts- und der Unsicherheitsausdrücke ist auch entscheidend: Wenn Faktizitätsausdrücke überwiegen, gibt es im gegebenen Diskurs feste, markante Positionen, die miteinander kontrastiert werden. Wenn aber die Unsicherheitsausdrücke dominieren, hat man den Eindruck, dass das ganze Thema einen ziemlich unsicheren Hintergrund hat.

\section{Qualitative Analyse}

Im letzten Analyseschritt werden exemplarische Textbeispiele ausgewählt, die für die sprachliche Realisierung eines epistemischen Hintergrundes besonders anschaulich sind. Diese werden unter Berücksichtigung der im 2. Kap. behandelten drei Analyseschwerpunkte qualitativ analysiert. Für den vorliegenden Beitrag wird ein Text ausgewählt, der beide Typen epistemischer Ausdrücke enthält, in dem jedoch die Faktizitätsausdrücke die leitende Rolle innehaben und die Unsicherheitsausdrücke untergeordnet sind. ${ }^{9}$

\footnotetext{
9 Die Faktizitätsausdrücke werden mit Fettdruck und Kursivschrift, die Unsicherheitsausdrücke nur mit Fettdruck hervorgehoben. Allgemeine Ausdrücke der Epistemik (hier drei sog. abtönungsfähige Partikeln, vgl. Weydt / Hentschel 1983) werden unterstrichen. Die Gliederung stammt von mir - A.P.
} 
Sie können davon ausgehen, dass wir uns über mehrere Staaten in Südostund Osteuropa Gedanken machen", heißt es in Brüsseler Kreisen. Namen will niemand nennen. Aber vielen scheint klar, dass dem in der vergangenen Woche geschnürten 20-Milliarden-Euro-Hilfspaket für Ungarn schnell weitere Unterstützung für andere EU-Länder folgen könnte.

„Wir wissen, dass auch andere Mitgliedstaaten und Nachbarn der Union unter Druck stehen“, stellt Wirtschaftskommissar Joaquín Almunia fest. Bulgarien etwa weist ein Leistungsbilanzdefizit von 25 Prozent aus. Rumänien und die baltischen Staaten gelten ebenfalls als Kandidaten für finanzielle Hilfe. Bislang allerdings sind keine weiteren Notrufe eingegangen. Die Union hat kein universelles Konzept, wie sie mit weiteren Krisen-Kandidaten umgehen wird. „Das entscheiden wir von Fall zu Fall“, erläutert die Sprecherin Almunias.

Über die Brüsseler Möglichkeiten zu helfen, befinden ohnehin die Mitgliedstaaten. Auch bei der von der EU-Kommission vorgeschlagenen Erhöhung ihres Spielraums für Zahlungsbilanzhilfen von zwölf auf 25 Milliarden Euro haben die nationalen Finanzminister das letzte Wort. Sie legen den Höchstbetrag fest, den die Kommission am Kapitalmarkt leihen kann, um das Geld Staaten in Not zur Verfügung zu stellen. Auf diesem Weg hat Brüssel 6,5 Milliarden Euro zum Hilfspaket für Ungarn beigesteuert und damit mehr als die Hälfte seiner derzeitigen Möglichkeiten ausgeschöpft. Bei ihrem heutigen Treffen werden die Finanzminister noch nicht über die Erhöhung des Spielraums entscheiden. Auch eine stärkere Einbeziehung der Europäischen Investitionsbank in Hilfsaktionen kann Brüssel zwar anregen und eine Kapitalerhöhung vorschlagen. Doch auch ein solcher Schritt steht heute nicht auf der Tagesordnung.

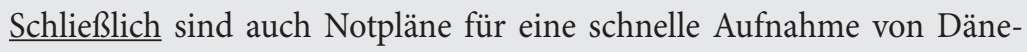
mark oder der baltischen Staaten in die Eurozone heute kein Thema. Mit Beitrittsabsichten wird in Brüssel aber fest gerechnet. Almunia: Bislang euroskeptische Staaten „erkennen, dass es in der Krise unbequemer wird, wenn man draußen ist."

Textbeispiel aus: Frankfurter Rundschau, 4.11.2008. 


\subsection{Die handlungsorientierte Analyse}

Im ersten einführenden Teil werden die Diskursakteure nur sehr vage bestimmt („Brüsseler Kreise“), die epistemischen Ausdrücke sind Unsicherheitsausdrücke: Gedanken machen und scheinen. Das ganze Thema, die Finanzhilfe der EU für finanzschwache südost- und osteuropäische Länder, wird am Anfang als ein schwieriges Thema mit vielen Unsicherheitsfaktoren dargestellt.

Dieser anfänglich unsichere Hintergrund verändert sich jedoch, wenn die Akteure konkretisiert werden. Im zweiten Teil, in dem der Wirtschaftskommissar Almunia sowie seine Sprecherin die aktuellen Diskursakteure sind, werden nur Faktizitätsausdrücke benutzt: wissen, feststellen, ausweisen, entscheiden, erläutern. Die beiden Akteure verkörpern zugleich auch die Europäische Kommission, besonders wenn sie in wir-Form sprechen. Die vorliegenden Faktizitätsverben drücken also die feste Position und die Entschlossenheit der Europäischen Kommission aus. Das Diskursobjekt, auf das sich diese Verben beziehen, ist einerseits die finanzielle Notsituation der Länder in Südost- und Osteuropa, andererseits die zu gewährende Finanzhilfe für sie. Implizit erscheinen also bereits in diesem ersten Teil auch andere Diskursakteure, die der Kommission gegenüberstehen. Diese Länder wollen aber die Hilfe der EU nicht in Anspruch nehmen. Mit Hilfe der abtönungsfähigen Partikel allerdings werden die beiden Parteien (Kommission vs. Länder in Südost- und Osteuropa) auch explizit einander gegenübergestellt.

Im dritten Teil verändern sich die Diskursakteure. Die Kommission steht hier den Mitgliedstaaten sowie ihrem Vertreter, dem Rat der Finanzminister, gegenüber. Das Ausdrucksmittel für diese Gegenüberstellung ist die exhaustiv-adversative Partikel ohnehin. ${ }^{10}$ Diesem Gremium kann die Kommission nur Vorschläge machen, die endgültigen Entscheidungen liegen bei den Finanzministern. Ausdrücke, deren Diskurssubjekt die Kommission ist, sind in diesem Textteil die Unsicherheitsausdrücke Möglichkeit (2mal), anregen und

\footnotetext{
10 Mit ohnehin wird markiert, dass zwei (oder mehrere) Möglichkeiten einander gegenübergestellt werden, aber letztendlich nur die einzige explizit ausgedrückte Möglichkeit gültig ist, die implizierten oder früher erwähnten ungültig erklärt werden.
} 
vorschlagen (2mal). ${ }^{11}$ Das Subjekt für die Faktizitätsverben befinden, festlegen und entscheiden sind die Finanzminister.

Im dritten Teil des Textes, der mit der Konsequenz ziehenden Partikel schließlich eingeleitet wird, stehen wieder die EU-Administration (in diesem Teil einfach Brüssel genannt) sowie die hilfsbedürftigen Staaten einander gegenüber. Die Position der EU-Administration wird mit dem Ausdruck fest rechnen charakterisiert, der sich zwar auf die Zukunft bezieht, aber einen hohen Sicherheitsgrad ausdrückt. Das andere epistemische Verb, erkennen, dessen Subjekt die hilfsbedürftigen Länder sind, drückt eine sich verändernde epistemische Einstellung aus. Ein Fakt, das bisher nicht bewusst war, wird erkannt. Damit wird hier auf die baltischen Staaten Bezug genommen, die die Entscheidungsgewalt der EU-Administration in der Zukunft annehmen werden und eine engere Beziehung zu Brüssel anstreben.

\subsection{Die gesellschaftsorientierte Analyse}

Die Diskursakteure positionieren sich mit den unterschiedlichen Handlungen bzw. mit dem Gebrauch der epistemischen Ausdrücke. Diese Positionen hängen eng mit den sozialen Stellungen der einzelnen Akteure im Diskurs, mit Über- und Unterordnungsverhältnissen, ja sogar mit Machtverteilungen zusammen. Diejenigen Akteure, die entscheiden, feststellen, festlegen oder befinden, sind in einer übergeordneten, dominierenden Position. Andere, die etwas vorschlagen oder erkennen (bzw. die wegen ihrer Finanzschwäche ausgeliefert sind), sind untergeordnet. Dadurch stellt sich heraus, dass die Kommission in Kontrast zu den hilfsbedürftigen Staaten eine dominante Position hat, den Mitgliedstaaten (die durch ihre Finanzminister vertreten sind) jedoch in diesem Diskurs untergeordnet ist (die Finanzminister verfügen über die letzte Entscheidung).

\footnotetext{
11 Es ist nämlich nicht sicher, dass die Kommission ihre Entscheidungen gegenüber dem Finanzministerrat durchsetzen kann.
} 


\subsection{Die wissensorientierte Analyse}

Der sich ständig verändernde epistemische Hintergrund hängt in hohem Maße von der Positionierung der einzelnen Diskursakteure ab. Solange selbst die Diskursakteure nur sehr vage definiert sind („Brüsseler Kreise“), sind auch die Positionen unsicher. Wenn die Kommission und der Wirtschaftskommissar in den Mittelpunkt gestellt werden, ändert sich dieser Hintergrund: Die Kommission zeigt eine sehr selbstsichere Position. Mit der wir-Form, mit der Aussage, dass sie über die wirtschaftliche Not bestimmter Länder Bescheid weiß und dass sie über Finanzhilfen entscheidet, wird angedeutet, dass sie eine klare Übersicht über die Krisensituation hat und zugleich in der Lage ist, die Krise entsprechend zu managen. In dem Punkt, in dem die Mitgliedstaaten (vertreten durch ihre Finanzminister) als Gegenakteure auftreten, wird diese feste Position der Kommission relativiert. Die Finanzminister, die „das letzte Wort“ haben, schränken die Position der Kommission ein, indem sie jetzt „noch nicht über die Erhöhung des Spielraums entscheiden“. Daraus folgt, dass die Finanzminister eine andere Perspektive haben und die Lage anders beurteilen. Es gibt also eine Spannung zwischen zwei Wissensperspektiven, die zugleich zwei Interessen verkörpern: das Interesse der Union auf der einen Seite und das Interesse der Mitgliedstaaten auf der anderen. Die Lösung ist die Erkenntnis der Notwendigkeit einer stärkeren Integration („erkennen, dass es in der Krise unbequemer wird, wenn man draußen ist"), wodurch eine Annäherung der beiden Interessen und auch der beiden Perspektiven möglich ist.

\section{Fazit}

Die epistemischen Ausdrücke bilden einen Rahmen für den ganzen Text: Der rahmenöffnende Ausdruck ist die auf einen unsicheren Hintergrund verweisende Kollokation Gedanken machen, der rahmenschließende ist das dynamisch-epistemische Verb erkennen. Im Laufe des Textes wird eine sehr klare, sichere Perspektive, die der Kommission, zunächst relativiert und mit anderen Perspektiven konfrontiert. Anschließend wird auch die Annäherung dieser Perspektiven als Lösung angedeutet, die durch eine neue Erkenntnis der Diskursakteure möglich ist. Die Diskursakteure können nämlich gerade durch die Krise zur Einsicht der Notwendigkeit einer engeren Integration kommen. 
In der vorliegenden Studie wurde gezeigt, dass eine methodenbewusste linguistische Analyse epistemischer Ausdrücke und deren Kombinationen bzw. Verteilungen in Diskursabschnitten vor dem theoretischen Hintergrund des beschriebenen diskurslinguistischen Modells es ermöglicht, Positionen und Perspektiven, Machtverhältnisse und die Entwicklung des gemeinsamen Wissens der Diskursakteure aufzudecken.

\section{Literatur}

Ágel, Vilmos / Hennig, Mathilde (2007): Überlegungen zur Theorie und Praxis des Nähe- und Distanzsprechens. In: Ágel, Vilmos / Hennig, Mathilde (Hrsg.): Zugänge zur Grammatik der gesprochenen Sprache. Tübingen: Niemeyer (RGL 269), 179-214.

Bubenhofer, Noah (2009): Sprachgebrauchsmuster. Korpuslinguistik als Methode der Diskurs- und Kulturanalyse. Berlin / New York: de Gruyter (Sprache und Wissen 4).

Busse, Dietrich (2008): Diskurslinguistik als Epistemologie. Das verstehensrelevante Wissen als Gegenstand linguistischer Forschung. In: Warnke, Ingo H. / Spitzmüller, Jürgen (Hrsg.): Methoden der Diskurslinguistik. Sprachwissenschaftliche Zugänge zur transtextuellen Ebene. Berlin / New York: de Gruyter, 57-87.

Felder, Ekkehard (2011): Pragma-semiotische Textarbeit und der hermeneutische Nutzen von Korpusanalysen für die linguistische Mediendiskursanalyse. In: Felder, Ekkehard / Müller, Marcus / Vogel, Friedemann (Hrsg.): Korpuspragmatik. Thematische Korpora als Basis diskurslinguistischer Analysen. Berlin / New York: de Gruyter (Linguistik - Impulse \& Tendenzen 44), 115-174.

Felder, Ekkehard (2013): Faktizitätsherstellung mittels handlungsleiternder Konzepte und agonaler Zentren. In: Felder, Ekkehard (Hrsg.): Faktizitätsherstellung in Diskursen. Die Macht des Deklarativen. Berlin / Boston: de Gruyter (Sprache und Wissen 13), 13-28.

Felder, Ekkehard (2016): Geschichte(n) zwischen Faktualität und Fiktionalität: Überlegungen zur Objektivierung von Ereignisnarrationen. In: Beßlich, Barbara / Felder, Ekkehard (Hrsg.): Geschichte(n) - faktual und fiktional. 
Literarische und diskursive Erinnerungen im 20. Jahrhundert. Frankfurt am Main: Peter Lang, 167-185.

Felder, Ekkehard / Müller, Marcus / Vogel, Friedemann (2011): Korpuspragmatik. Paradigma zwischen Handlung, Gesellschaft und Kognition. In: Felder, Ekkehard / Müller, Marcus / Vogel, Friedemann (Hrsg.): Korpuspragmatik. Thematische Korpora als Basis diskurslinguistischer Analysen. Berlin / New York: de Gruyter (Linguistik - Impulse \& Tendenzen 44), 3-30.

Péteri, Attila (2018): Epistemik und Faktizität in Pressediskursen. In: Bartoszewicz, Iwona / Szczęk, Joanna / Tworek, Artur (Hrsg.): Grenzen der Sprache - Grenzen der Sprachwissenschaft. Bd. II. Wrocław / Dresden: Neisse, 27-38.

Warnke, Ingo H. / Spitzmüller, Jürgen (2008): Methoden und Methodologie der Diskurslinguistik. Grundlagen und Verfahren einer Sprachwissenschaft jenseits textueller Grenzen. In: Warnke, Ingo H. / Spitzmüller, Jürgen (Hrsg.): Methoden der Diskurslinguistik. Sprachwissenschaftliche Zugänge zur transtextuellen Ebene. Berlin / New York: de Gruyter (Linguistik - Impulse \& Tendenzen 31), 3-54.

Weydt, Harald / Hentschel, Elke (1983): Kleines Abtönungswörterbuch. In: Weydt, Harald (Hrsg.): Partikeln und Interaktion. Tübingen: Niemeyer, 3-24. 